TARNOWSKIE STUDIA TEOLOGICZNE 36 (2017) NR 2, S. 61-74

http://dx.doi.org/10.15633/tst.2615

ks. Sławomir Jeziorski ${ }^{1}$

WYŻSZE SEMINARIUM DUCHOWNE W RZESZOWIE

\title{
La definizione di sacramento in pensiero di Pietro Lombardo
}

Lo scopo del presente studio è quello di offrire un approccio al concetto di sacramento nel pensiero di Pietro Lombardo. Tale argomento sembra aver il suo valore particolare perché, come afferma Enrico Mazza, „l'importanza di Lombardo nella storia della teologia è straordinaria, dato che tutti i grandi scolastici hanno una dipendenza più o meno stretta con questo autore" ${ }^{\text {"2 }}$ Occorre ancora aggiungere che l'argomento trattato non tenta di fare una comparazione fra il pensiero del nostro autore e gli altri teologi, sia quelli precedenti che quelli contemporanei a Pietro Lombardo. Anzi, l'esame che sta per essere fatto prende in considerazione la famosa definizione di sacramento scritta nelle Sententiae, cercando di esporla e comprenderla nel proprio contesto storico-culturale e teologico. Questo studio ci permette di valutare adeguatamente il valore della definizione con i suoi punti forti e deboli. Tale proseguimento determina anche la struttura dello studio. All'inizio, sarà presentato brevemente il contesto in cui scriveva l'autore esaminato. Dopo di che, verranno messi in luce i singoli elementi della definizione da lui fatta, per fornire, alla fine, la prova di valutazione del concetto del sacramento.

1 Don Sławomir Jeziorski - il presbitero della diocesi di Rzeszów (Polonia), dottore nella liturgia, laureatosi nel 2017 presso il Pontificio Istituto Liturgico di Sant’Anselmo a Roma, professore di liturgia nel Seminario Maggiore a Rzeszów e l’insegnante della religione nel Liceo Classico di San Giovanni Paolo II della stessa città.

2 E. Mazza, Elementi agostiniani necessari per la concezione sacramentale della liturgia, in: Per ritus et preces. Sacramentalità della liturgia. Atti viII Congresso Internazionale di Liturgia (Roma, Pontificio Istituto Liturgico, 16-18.05.2007), a cura di P. A. Muroni, Roma 2010, p. 69. 


\section{Il contesto storico-culturale e teologico}

Non è possibile comprendere bene il concetto di sacramento nella teologia di Pietro Lombardo, senza collocarlo nel contesto in cui l'autore pensava e scriveva la sua teologia. Prescindendo da tale procedimento, rischiamo di fraintendere la teologia che stiamo per esaminare. Occorre dire, però, che non è possibile presentare qui una visione dettagliata dell'epoca di Pietro Lombardo e non cerco di farlo, essendo consapevole che la presentazione del contesto svolge solo una funzione secondaria, in questo lavoro. Questo limite non cambia, però, il fatto che, nel dodicesimo secolo sono stati i fattori che hanno influito sul pensiero dei teologi medievali.

Fra quelli che sembrano essere più notevoli, ci si può all'inizio fermare alle trasformazioni della struttura sociale del popolo. In questa epoca „suscitano le nuove classi sociali costituite dai mercanti e dagli artigiani con il suo individualismo devozionale" ${ }^{3}$. Tale cambiamento che, a prima vista, non influisce sulla teologia, trasforma la mentalità del popolo che con la sua devotio christiana sta allontanandosi dalla liturgia.

Di conseguenza, nel confronto con questa devotio christiana „si può notare una corrente spirituale la quale è preoccupata di attuare una liturgia

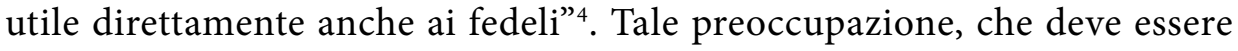
considerata come una reazione alla situazione religiosa, porta un arricchimento di elementi capaci di suscitare la sensibilità dei fedeli dinanzi al mistero cristiano. Una opinione del tempo esprime: "Ciò che i laici possono conoscere e può essere detto a loro di quanto riguarda la messa, può essere compreso in tre capi, ossia le vesti, i gesti, la pronuncia di parole"s. In seguito alla crescita degli elementi sensibili, veniva anche la necessità di spiegarli. Tali spiegazioni hanno assunto un metodo allegorico in cui ogni elemento aveva il suo riferimento al mistero celebrato. La liturgia diventava, quindi, uno spettacolo con molti elementi simbolici, ma d'altra parte con tante confusioni per quanto riguarda la distinzione di ciò che è fondamentale e ciò che deve essere solo come un ornamento.

3 E. Cattaneo, Il culto cristiano in Occidente. Note storiche (Biblioteca Ephemerides Liturgicae. Subsidia, 13), Roma 1992, p. 213.

4 E. Cattaneo, Il culto cristiano in Occidente..., p. 214.

5 E. Cattaneo, Il culto cristiano in Occidente..., p. 218. 
Un altro cambiamento importante dal nostro punto di vista è quello della comprensione del concetto di segno. Può darsi che anche a motivo di tanti segni aggiunti alla liturgia, il segno non viene più compreso nel modo in cui fu compreso dai Padri. „Se nell'antichità cristiana la mentalità biblica e la tradizione platonica avevano ispirato la creazione e la comprensione del simbolismo liturgico cristiano, nel Medioevo la perdita progressiva di un contatto vivo con la sacra Scrittura e il prevalere di indirizzi teologici intellettualistici ed astratti hanno favorito un'interpretazione allegorica e moralistica dei simboli cristiani"6. In altre parole, l'epoca medievale, usando lo stesso concetto di segno, lo comprende in modo diverso e cade „nell'impossibilità di capire che un simbolo (segno) è anche realtà" 7 .

Riassumendo, si può affermare che i teologi del Medioevo si trovarono nella situazione in cui i sacramenti erano celebrati con tanta drammaturgia, assomigliandoli agli spettacoli e con il simbolismo che non avendo già la sua comprensione patristica, veniva spiegato attraverso l'allegoria. Dall'altra parte suscitavano le prove di ritrovare la comprensione dei sacramenti e darne una definizione. Così entriamo nel pensiero di Pietro Lombardo.

\section{Il concetto di sacramento di Pietro Lombardo}

Pietro Lombardo dedica ai sacramenti il quarto libro delle sue sentenze . $^{8}$ In esso fa una introduzione in cui appare la sua definizione del sacramento e, in seguito alla chiave presentata, descrive i sette sacramenti. Occorre, all'inizio, richiamare il testo stesso di Lombardo che sta alla base di questo lavoro. Esso si presenta nel modo seguente:

Sacramentum enim proprie dicitur, quod ita signum est gratiae Dei et invisibilis gratiae forma, ut ipsius imaginem gerat et causa exsistat. Non igitur significandi tantum gratia sacramenta instituta sunt, sed et sanctificandi ${ }^{9}$.

6 D. Sartore, Segno/simbolo, in: Liturgia, a cura di D. Sartore, A. M. Triacca, C. Cibien, Cinisello Balsamo 2001, p. 1859.

7 B. Neunheuser, Storia della liturgia attraverso le epoche culturali (Biblioteca Ephemerides Liturgicae. Subsidia, 11), Roma 19993, p. 118.

8 Petrus Lombardus, Sententiae in IV libris distinctae. Tomus 2: Liber 3 et 4, Grottaferrata 1981.

9 Petrus Lombardus, Sententiae..., Lib. 4, dist. 1, cap. 4,2. 


\section{L'analisi letteraria}

Già la prima lettura e l'analisi letteraria mostrano la quadruplice struttura del testo. Nella definizione, possiamo individuare la prima parte che consiste nella proposizione principale: „Sacramentum [...] ita singum est gratiae Dei et invisibilis gratiae forma”. Questa frase contiene due affermazioni su ciò che è il sacramento: signum gratiae Dei come pure invisibilis gratiae forma. Poi Lombardo usa la proposizione finale che descriva la funzione del sacramento compreso come il segno e la forma. Questa proposizione viene così esperssa: ut ipsius imaginem gerat et causa exsistat. Anche qui possiamo distinguere due proposizioni: ut immaginem gerat come pure ut causa exitstat. La definizione presenta, quindi, il duplice scopo del sacramento, compreso come il segno e la forma: portare l'immagine ed essere la causa.

È da notare ancora che il soggetto comune di tutte e quttro le parti è la gratia. Infatti la definizione parla di: segno gratiae, forma gratiae, imago ipsius (=gratiae) e causa ipsius (=gratiae). Si può riassumere che l'autore „si concentra sull'idea di grazia in ciascuno dei quattro aspetti che riconosce nel sacramento" ${ }^{10}$. Tale grazia, vista nel contesto dato, può essere considerata come il rimedio per i peccati, secondo le parole di Pietro Lombardo il quale scrive: "quia contra peccati originalis et actualis vulnera sacramentorum remedia Deus instituit"11. L'autore delle Sentenze in questo momento non si ferma su che cosa è la grazia, ma entra direttamente nel concetto di segno che è fondamentale per le sue analisi. Lo seguiamo.

\section{Il concetto del segno}

I'importanza che Lombardo dà al concetto di sengo si può notare già dall'inizio della sua teologia dei sacramenti. Infatti, tutta questa parte della sua opera viene titolata De Doctrina Signorum. Lombardo tiene fede alla linea teologica dell'epoca in cui „la sacramentalità è sempre descritta in termini

${ }^{10} \mathrm{D}$. Van Den Eynde, Les définitions des sacrements pendant la première période de la théologie scolastique, "Antonianum" 34 (1949), s. 223.

${ }_{11}$ Petrus Lombardus, Sententiae..., Lib. 4, dist. 1, cap. 1. 
agostiniani come «segno»"12. Allora, lasciando a parte il concetto di sacrum secretum, egli passa subito alla nozione signum, facendone una premessa per la definizione del sacramento. Così „Pietro integra la sua definizione del sacramento con la teoria dei segni e delle cose, di Agostino" ${ }^{13}$. Di conseguenza, l'autore delle Sentenze scrive che il segno è la cosa che oltre l'aspetto che si presenta ai sensi, porta alla conoscenza qualcos'altro fuori di sé ${ }^{14}$. Seguendo sant'Agostino ${ }^{15}$, il teologo medioevale va avanti e fa una divisione. Questo ha la sua importanza, perché „se infatti i teologi o i canonisti precedenti, tutt'al più avevano menzionato la definizione di segno, senza chiarire bene in che senso e in che modo esso serviva al piano generale di un trattato sui sacramenti, Pietro Lombardo non solo cita questa definizione, ma procede riprendendo anche la classificazione più generale dei segni proposta da Agostino per specificare in quale dei due rami principali (signa naturalia vs. signa data) vanno collocati i sacramenti del Nuovo Testamento"16. Così il nostro autore fa una distinzione che in modo chiaro permette di collocare i sacramenti in tale teoria del segno.

Il primo gruppo, richiamato da Agostino, consiste nei segni naturali, il secondo invece, in quelli dati. I segni naturali sono quelli che vengono dalla natura, quelli dati, invece, essendo determinati dagli uomini, possono essere chiamati i segni convenzionali. Dopo questa prima divisione, l'autore delle Sentenze specifica dentro i segni convenzionali quelli che sono dei sacramenti e quelli che non lo sono. Il sacramento consiste, quindi come il segno dato, particolare. Schematicamente la sistematizzazione fatta da Lombardo si presenta nel modo seguente:

${ }_{12}$ E. Mazza, Continuità e discontinuità. Concezioni medievali delleucaristia a confronto con la tradizione dei Padri e della liturgia (Biblioteca Ephemerides Liturgicae. Subsidia, 113), Roma 2001, s. 157.

${ }_{13}^{13}$ M. Colish, Peter Lombard, Leiden-New York-Köln 1994, p. 528.

${ }^{14}$ Il testo latino lo esprime: "Signum vero est res, praeter speciem quam integrit sensibus, aliud aliquid ex faciens in cogitationem venire" (Petrus Lombardus, Sententiae..., Lib. 4, dist. 1, cap. 3).

${ }^{15}$ Augustinus, De doctrina christiana, ed. J. Martin (Corpus Christianorum. Series Latinae, 32), Turnhout 1962, Lib. 2, cap. 1-2.

${ }^{16}$ C. Marmo, Segno e immagini nelle Sententiae di Pietro Lombardo, in: Pietro Lombardo. Atti dell XLIII Convegno storico internazionale (Todi, 8-10.10.2006), a cura di E. Menestò, Spoleto 2007 , p. 52. 
SIGNUM

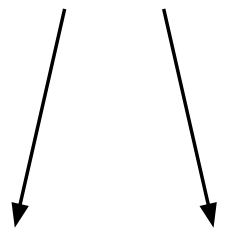

NATURALE - DATUM

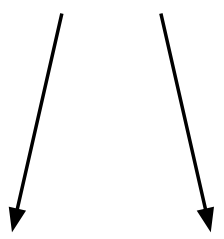

SACRAMENTUM - NON SACRAMENTUM

Dopo aver fatto questa distinzione che colloca i sacramenti nel gruppo dei segni convenzionali, occorre fermarsi sul concetto stesso del segno. Pietro lo definisce, seguendo Agostino, come res che può far venire in mente qualcos'altro, oltre sé. Per Pietro Lombardo, res non ha un significato univoco. Res viene intesa come l'oggetto distaccato dalla realtà significata. Questo risulta dal testo stesso delle Sentenze che, riguardo l'Eucaristia, afferma: „Sed quia corpus Christi incorruptibile est, sane dici potest fractio illa et partitio non in substantia corporis, sed in forma panis sacramentaliter fieri: ut vera fractio et partitio sit ibi, quae fit non in substantia, sed in sacramento, id est in specie" ${ }^{17}$. Il segno viene, quindi compreso tramite i termini della filosofia aristotelica e il sacramento diventa solo una cosa che viene in mente, fa un ricordo della realtà, ma non la contiene necessariamente. Quando viene spezzato il pane, viene spezzato solo il segno, non la realtà stessa. Così si pone una domanda. Se il segno è soltanto un oggetto, una cosa che porta nella mente una realtà, esso ha la sua efficacia? Per rispondere a tale domanda, Pietro introduce il concetto di causa.

${ }_{17}$ Petrus Lombardus, Sententiae..., Lib. 4, dist. 4, cap. 3,2-3. 


\section{La causalità}

Il concetto di causa è una cosa particolare attribuita al nostro Autore. Infatti, „la maggior novità della teologia sacramentaria di Pietro Lombardo consiste nell'aver definito i sacramenti, per la prima volta, come causa della grazia” ${ }^{\prime 8}$. Lombardo usa „il concetto comune di «causa» per esprimere l'idea dell'efficacia del sacramento" ${ }^{19}$ cioè per spiegare in che senso il sacramento sia segno. Dobbiamo tener conto che l'epoca in cui scriveva il Maestro si caratterizzava per l'aumento delle tante celebrazioni della devotio christiana, come pure dei tanti segni spiegati in modo allegorico. Esisteva, pertanto, una esigenza di chiarire in che senso il sacramento possa essere considerato come il segno. Il tentativo di Pietro Lombardo va nella linea di aggiungere il concetto di causalità. Nelle sue Sentenze, „il sacramento è descritto come segno, e la categoria della causa viene introdotta per spiegare l'efficacia sacramentale" ${ }^{20}$. In certo, senso si può intuire che tale formulazione in modo esplicito esprime ciò che nell'epoca patristica era una cosa naturale. Per i Padri il segno, detto anche simbolo, era una realtà efficace che rendeva presente tutto il mistero celebrato. Dopo l'eresia di Berengario, il segno ha perso la sua forza, e nell'epoca di numerosi segni ornamentali, tale aggiunta era necessaria. Resta ancora la domanda da dove venga la forza di tale efficacia. Il Maestro offre una soluzione, quando risponde alla domanda, perché non furono istituiti i sacramenti immediatamente dopo il peccato originale. A questo punto, egli afferma che il motivo è che i sacramenti ricevono e danno la grazia che nasce dalla passione di Cristo ${ }^{21}$. I sacramenti sono, quindi, i segni efficaci della grazia, in modo tale che la grazia deriva dall'opera salvifica di Cristo.

${ }^{18}$ E. Ruffini, Sacramenti, in: Dizionario teologico interdisciplinare, a cura di L. Pacomio, vol. 3, Torino 1977, p. 187.

${ }^{19}$ E. Mazza, Elementi agostiniani necessari per la concezione sacramentale della liturgia, p. 70 .

${ }^{20}$ E. Mazza, La celebrazione eucaristica. Genesi del rito e sviluppo dell'interpretazione, Bologna 2003, p. 220.

${ }^{21}$ Cfr. J. M. Sierra López, El número septenario de los sacramentos, in: Asociación Española de Profesores de Liturgia, La sacramentalidad de la liturgia,, Barcelona 2010, p. 120. 


\section{La somiglianza}

Dopo aver affermato che il sacramento è il segno che causa la grazia, Pietro Lombardo doveva risolvere un'altra difficoltà. Essa consisteva nella domanda, quale fosse la caratteristica che individua i sacramenti dagli altri segni convenzionali. Egli dà la risposta scrivendo: „Sacramentum eius rei similitudinem gerit, cuius signum est" ${ }^{22}$. Tale affermazione è basata sulla frase di Agostino, che il Maestro cita, appartando qualche cambiamento: „Si enim sacramenta non haberent similitudem rerum quarum sacramenta sunt, proprie sacramenta non dicerentur" ${ }^{23}$. Senza entrare nel testo agostiniano, ci fermiamo al concetto di similitudo, che, per Lombardo, è distintivo per quanto riguarda i sacramenti.

La somiglianza nei sacramenti si riferisce all'aspetto visibile del segno. Essa, però „non viene intesa come banale somiglianza, ovvero come possesso di una medesima qualità o altro accidente, ma va piuttosto interpretata come analogia o proporzione, ovvero come somiglianza tra le relazioni che gli oggetti in questione intrattengono con uno o più altri oggetti”24. Per chiarire si può richiamare un esempio sull'Eucaristia. Enrico Mazza lo spiega: „in Pietro Lombardo, dunque, la similitudo expressa appartiene alla «natura» delle specie eucaristiche, in quanto visibili, e non alla «natura» del sacramento in quanto tale; ossia appartiene all'area del segno e non all'area della res" 25 .

Tale somiglianza Lombardo la vede nel segno naturale, come abbiamo potuto vedere all'esempio del pane e dell'Eucaristia. Ma occorre aggiungere, che "la relazione di somiglianza o di analogia non ne fa automaticamente un segno naturale, ma piuttosto rende manifeste le ragioni (o i rispetti) per cui un certo oggetto è stato scelto da Dio come elemento materiale o res del sacramento" 26 . Dio, infatti, è colui che, secondo Lombardo, ha istituito i sacramenti e, di conseguenza, ha scelto i segni naturali, facendone i segni efficaci della grazia. Tale segno sacramentale assume la propria forma e su di essa dobbiamo fermarci adesso.

${ }^{22}$ Petrus Lombardus, Sententiae..., Lib. 4, dist. 1, cap. 4,1.

${ }^{23}$ Cfr. Augustinus, Epistola 98, 9, in: Epistulae LVI-C, ed. K. D. Daur (Corpus Christianorum. Series Latinae, 31A), Turnhout 2005.

${ }^{24}$ C. Marmo, Segno e immagini nelle Sententiae di Pietro Lombardo, p. 83.

${ }^{25}$ E. Mazza, Continuità e discontinuità..., p. 157.

${ }^{26}$ C. Marmo, Segno e immagini nelle Sententiae di Pietro Lombardo, p. 83. 


\section{La forma di sacramento}

Trattando la forma occorre tener presente il cambiamento della comprensione di questa parola menzionato da Enrico Mazza. Egli scrive: „è possibile che il Maestro sia tributario della accezione primitiva di questo termine, in uso nell'alta scolastica, quando «forma» designava la «maniera» di compiere il rito nel suo complesso. In questo uso arcaico, il termine forma avrebbe la stessa funzione di tipo. Successivamente il termine forma disegna alcune parole specifiche e determinate, che sono la "formula» sacramentale" ${ }^{\text {"27 }}$. Questa citazione in breve parole descrive non solo il significato del concetto di forma, ma anche il cambiamento della sua comprensione durante il secoli. Lasciando a parte il processo di mutamento del concetto occorre affermare che per Lombardo la forma è la forma sacramentale, cioè alcune parole che devono essere pronunciate insieme con il gesto perché possa essere compiuto il sacramento.

\section{Excursus: Il settenario}

Vale la pena aggiungere che tale definizione ha permesso a Pietro Lombardo di fare una distinzione fra i sacramenti in senso stretto e gli altri riti, sia quelli dell'Antico Testamento, sia quelli che fiorivano nella devotio cristiana del tempo. Lautore delle sentenze, per fare questa distinzione, usa la sua definizione del sacramento e soprattutto il concetto di causa. La linea di condivisione viene dal fatto che i sacramenti del Nuovo Testamento non solo significano la realtà, ma ne portano la grazia, cioè, usando le parole di Lombardo: „haec autem dant salutem ${ }^{\text {"28 }}$. Dopo aver affermato tale distinzione, Teologo scrive ciò che deve essere considerato come sacramento: „Iam ad sacramenta novae Legis accedamus: que sont baptismus, confirmatio, panis benedictionis, id est eucharistia, poenitentia, unctio extrema, ordo, coniugium"29. Occore, però, notare, che questo non è una creazione pura e sempllice dei teologi. Si può, invece, affermare: „se, nel xII secolo, si determina la lista canonica dei sacramenti, è perché, nellesperienza ecclesiale, questi gesti ecclesiali esprimomo tradizionalmente la fede e il dono divino

\footnotetext{
${ }^{27}$ E. Mazza, Continuità e discontinuità..., p. 160.

${ }^{28}$ Petrus Lombardus, Sententiae..., Lib. 4, dist. 1, cap. 6.

${ }_{29}$ Petrus Lombardus, Sententiae..., Lib. 4, dist. 2, cap. 1,1.
} 
fatto alla Chiesa" ${ }^{30}$. L'autore delle sentenze deve essere, pertanto, considerato più come il redattore che il creatore del settenario.

\section{La valutazione del concetto di sacramento}

Dopo aver presentato il concetto di sacramento in Pietro Lombardo, occorre provare a fare una valutazione di questo pensiero. In questo compito bisogna considerare il contesto in cui la definizione fu creata. Nel Medioevo si notava la fioritura delle diverse forme della devozione, l'aumento dei segni mal compresi e mai interpretati. Tale confusione esigeva di fare uno chiarimento e una distinzione fra i segni che hanno avuto solo una funzione di ornamento e quelli che realmente mettevano i partecipanti in contatto con la realtà divina. Fra le diverse definizioni fatte in quest'epoca, quella di Pietro Lombardo deve essere considerata come una prova di compire tale compito. L'introduzione del concetto di causalità ha tentato di recuperare la forza efficace del segno che è andata persa dopo l'eresia di Berengario. Questo sicuramente è il valore del pensiero lombardiano.

D'altra parte, però, occorre notare la definizione che ha dato una chiarimento, ha messo il sacramento nei quadro dei concetti astratti, distaccandoli dalla storia della salvezza. Infatti, ,identificando gli elementi essenziali del sacramento con la materia e la forma, il fatto sacramentale perderà la sua caratteristica fondamentale di «azione» e di «avvenimento», per cui l'interesse della teologia e della catechesi si sposterà, dal mistero e dalle persone che ne sono coinvolte, alle cose con cui il mistero è celebrato" ${ }^{31}$.In altre parole, la mentalità creata sulla base del pensiero scolastico di cui Lombardo è rappresentante, „la celebrazione si riduce ad essere una sorta di enfatizzazione di ciò che è ritenuto essenziale nella concezione ilemorfica di sacramento"32. Di conseguenza, il smarrimento del linguaggio simbolico ha esposto „il rito al rischio d'essere inteso unicamente come esteriorità da cui difendersi se si vuol salvaguardare veramente la possibilità di un reale incontro con Dio"33.

${ }^{30} \mathrm{H}$. Bourgeols, I segni della salvezza vol. 3: 12-20 secolo: sacramenti e Chiesa, Vergine Maria, Casale Monferrato 1998, p. 119.

${ }^{31}$ E. Ruffini, Sacramenti, p. 188.

${ }^{32}$ F. Brovelli, La prassi liturgica nelle diverse epoche culturali, in: Celebrare il mistero di Cristo, vol. 2: La celebrazione dei sacramenti, a cura di G. Bonaccorso, Roma 1996, p. 196.

33 F. Brovelli, La prassi liturgica nelle diverse epoche culturali, p. 196. 
Bisogna tener conto che nella definizione manca qualsiasi riferimento a Cristo e alla Chiesa. Nelle Sentenze „la concezione del sacramento risulta ormai elaborata in un contesto più individuale, dove la «grazia» operata efficacemente dal sacramento, si configura come la grazia data, aumentata, ridata all'individuo" ${ }^{34}$. Mancano le categorie della partecipazione, l'evento, la celebrazione. Tutto sembra essere contenuto nella linea segno-distribuzione-uomo. Vale la pena aggiungere che tutto questo procedimento viene messo nel contesto del rimedio dei peccati in cui sacramenti sono collocati.

Riassumendo la definizione indubbiamente ha il suo valore e aveva la sua importanza nel tempo in cui fu creata. Ha permesso di ordinare la materia sacramentale nel momento di confusione, ma essendo il frutto di una epoca e un pensiero teologico contiene anche i punti deboli.

\section{Conclusione}

Il secolo xx ci ha offerto molta ricchezza teologica. Il rinnovamento biblico, patristico e liturgico ha portato la nuova freschezza teologica. Bisogna menzionare la riscoperta del Mistero Pasquale, la storia della salvezza, il concetto del simbolo e tanti altri. Tutto questo si incontra con il postmodernismo in cui la Chiesa vive e svolge la sua missione. Essendo consapevole che la mentalità della gente viene imbevuta di individualismo, la solitudine sperimentata, la difficoltà di pensare costruttivamente e l'incapacità di comprendere i concetti metafisici, la definizione di Pietro Lombardo sembra non sufficiente.

Con rispetto adatto alla tradizione, occorre però, a mio parere, esprimere la realtà sacramentale nel modo più comprensibile per la gente. Ereditando il concetto di segno, sembra necessario esprimerlo in tale modo che sia il mezzo per entrare nel rapporto personale con il Cristo Pasquale Che vive nella sua Chiesa. Bisogna ricuperare l'importanza della celebrazione dei sacramenti e, di conseguenza, mettere il rilievo la dimensione ecclesiale di tale celebrazioni.

La stanca cultura europea ha bisogno della vita nuova, della speranza nuova e tutto questo la Chiesa lo può offrire. Rimane allora urgente la necessità

${ }^{34}$ A. Caprioli, La riflessione teologica sui sacramenti in età medioevale, in: Celebrare il mistero di Cristo, vol. 2, p. 292. 
di trasmettere la ricchezza della via divina nel linguaggio compreso nel nostro tempo. Questo è il compito da assolvere.

\section{Bibliografia}

Augustinus, De doctrina christiana, ed. J. Martin (Corpus Christianorum. Series Latinae, 32), Turnhout 1962.

Bourgeols H., I segni della salvezza, vol. 3: 12-20 secolo: sacramenti e Chiesa, Vergine Maria, Casale Monferrato 1998.

Brovelli F., La prassi liturgica nelle diverse epoche culturali, in: Celebrare il mistero di Cristo, vol. 2: La celebrazione dei sacramenti, a cura di G. Bonaccorso, Roma 1996, p. 172-228.

Caprioli A., Alle origini della „definizione” di sacramento, „La scuola cattolica” 102 (1974), p. 718-743.

Caprioli A., La riflessione teologica sui sacramenti in età medioevale, in: Celebrare il mistero di Cristo, vol. 2: La celebrazione dei sacramenti, a cura di G. Bonaccorso, Roma 1996, p. 281-301.

Cattaneo E., Il culto cristiano in Occidente. Note storiche (Biblioteca Ephemerides Liturgicae. Subsidia, 13), Roma $2003^{2}$.

Colish M., Peter Lombard, Leiden-New York-Köln 1994.

Marmo C., Segno e immagini nelle "Sententiae” di Pietro Lombardo, in: Pietro Lombardo. Atti del XLIII Convegno storico internazionale (Todi, 8-10.10.2006), a cura di E. Menestò, Spoleto 2007, p. 51-88.

Mazza E., La celebrazione eucaristica. Genesi del rito e sviluppo dell'interpretazione, Bologna 2003.

Mazza E., Continuità e discontinuità. Concezioni medievali delleucaristia a confronto con la tradizione dei Padri e della liturgia (Biblioteca Ephemerides Liturgicae. Subsidia, 113), Roma 2001.

Mazza E., Elementi agostiniani necessari per la concezione sacramentale della liturgia, in: Per ritus et preces. Sacramentalità della liturgia. Atti vin Congresso Internazionale di Liturgia (Roma, Pontificio Istituto Liturgico, 16-18.05.2007), a cura di P. A. Muroni, Roma 2010, p. 45-86.

Neunheuser B., Storia della liturgia attraverso le epoche culturali (Biblioteca Ephemerides Liturgicae. Subsidia, 11), Roma $1999^{3}$.

Nielsen O. L., Peter Lombard in the Teological Context of the 12th Century: the Theological Virtue of Charity, in: Pietro Lombardo. Atti dell XLIII Convegno 
storico internazionale (Todi, 8-10.10.2006), a cura di E. Menestò, Spoleto 2007, p. 411-432.

Petrus Lombardus, Sententiae in IV libris distinctae, tomus II: Liber III et IV, Grottaferrata 1981.

Ruffini E., Sacramenti, in: Dizionario teologico interdisciplinare, a cura di L. Pacomio, vol. 3, Torino 1977, p. 184-201.

Sartore D., Segno/simbolo, in: Liturgia, a cura di D. Sartore, A. M. Triacca, C. Cibien, Cinisello Balsamo 2001, p. 1853-1864.

Sierra López J. M., El número septenario de los sacramentos, in: Asociación Española de Profesores de Liturgia, La sacramentalidad de la liturgia, Barcelona 2010, p. 109-141.

Van Den Eynde D., Les définitions des sacrements pendant la première période de la théologie scolastique, „Antonianum” 34 (1949), p. 183-228.

\section{Riassunto}

Le Sentenze di Pietro Lombardo influirono molto sul pensiero teologico medievale. Di conseguenza non è priva del significato per la teologia la sua definizione del sacramento, la quale si trova nell'opera principale del autore. Presente articolo è un tentativo di descrivere quella definizione che diede una base per la sistematizzazione e la definizione del settenario dei sacramenti nella Chiesa cattolica. A tale scopo, prima è stato brevemente presentato il contesto storico-culturale del pensiero di Lombardo. Poi, su quello sfondo, è stata presentata la definizione del sacramento nelle Sentenze. Essa è stata sottomessa all'analisi grammatica e successivamente a quella teologica. E questa seconda contiene la descrizione del segno, casualità, somiglianza e forma del sacramento.

\section{Parole chiavi}

Pietro Lombardo, liturgia, sacramento, somiglianza, segno, causalità

\section{Summary}

\section{The Definition of "Sacrament" in Peter Lombard's Thought}

The Sentences of Peter Lombard had a big influence on medieval theological thought. So, Lombard's definition of the sacrament is not without meaning for theology. 
This article is an attempt to explain the concept that became a basis of systematisation of the seven sacrament of the Catholic Church. For this reason, the article begins with the historical-cultural context of Lombard's ideas. On this landscape, the definition of a sacrament is examined, starting with literal analysis along with theological insights. The theological explanations contains a description of sign, causality, similarity and form of sacrament.

\section{Keywords}

Peter Lombard, liturgy, sacrament, similarity, sing, causality, form 